\title{
Charge state of silver halide colloids determines the antibacterial activity in amorphous calcium phosphate
}

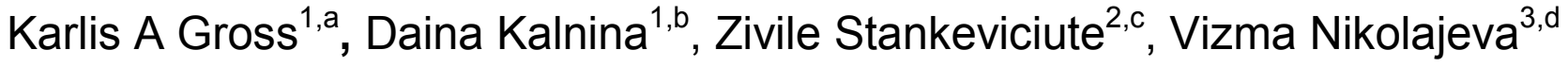 \\ ${ }^{1}$ Institute of Biomaterials and Biomechanics, Riga Technical University, Latvia \\ ${ }^{2}$ Faculty of Chemistry, University of Vilnius, Lithuania \\ ${ }^{3}$ Faculty of Biology, University of Latvia, Latvia \\ akgross@rtu.lv (corresponding author), bdaina.kalnina@rtu.Iv, 'zivile.stankeviciute@chf.vu.lt \\ dvizma.nikolajeva@lu.Iv
}

Keywords: antimicrobial, calcium phosphates, silver iodide, hydroxyapatite, bone implants

\begin{abstract}
Removal of bacteria is important not only at implantation, but after long-term implant/prosthesis use. This requires strategies that employ different approaches for combating bacteria. Halides have the potential of an additional mechanism, and together with silver may provide a more powerful antibacterial strategy. Silver iodide was synthesized as colloids with a positive and negative charge and incorporated into an amorphous calcium phosphate (ACP) to provide a possible greater antibacterial action. Colloids were characterized by FTIR spectroscopy and the charge measured by zeta potential. Phase analysis by X-ray diffraction patterns confirmed the formation of $\beta$-AgI nanoparticles. Minimum inhibitory concentrations (MIC) for preventing the growth of Staphylococcus aureus and Pseudomonas aeruginosa were lower for ACP containing negatively charged silver halides. Amorphous calcium phosphates with silver iodide exhibited good inhibition capacity. Solubility was determined by the increase in $\mathrm{pH}$ and the release of silver after 48 hours. The minimum bactericidal concentration (MBC) was also determined. This work has shown the effect of AgI charge in amorphous calcium phosphate for providing antibacterial action.
\end{abstract}

\section{Introduction}

Antimicrobial resistance is becoming a priority with implantable devices as an additional functionality to maintain implant integration within the body. Increasing measures are being taken to ensure sterility within the operating theatres in addition to sterilization of implants by ethylene dioxide or gamma irradiation. An additional means of providing antibacterial activity is to include agents that can fight off undesirable bacteria. These may be drugs that need to be added into a resorbable layer or ionic species integrated into the biomaterial structure.

Silver is receiving more interest since it is known to provides the most powerful bacteria combating action. Inclusion of silver into the apatite lattice during crystallization of calcium phosphates in solution is limited due to the larger size of the atomic species. Other means of incorporating silver involves the inclusion of silver phosphates or silver carbonate. Silver phosphate may be initially added or form alongside silver oxide during high temperature heating for scaffold or porous particulate production.

If silver is added as a nanoparticulate additive, then the chemistry may be improved by including other species capable of fighting bacteria. Silver iodide contains two chemical species that are known to separately provide antibacterial activity. Furthermore, silver iodide (AgI) can be prepared as a charged sol by increasing the concentration of silver ions or iodide on the sol surface, Figure 1. The negatively charged sol - labeled as $\mathrm{AgI}^{-}$for simplicity - can be viewed as AgI particles with an excess of iodide ions. The concentration of ions is not limited by the need to retain stoichiometry within the crystal, but is set by the solution conditions used to synthesize the sol. Consequently, the use of charged AgI sols can adjust the size, concentration, and charge to increase the antimicrobial activity. 
Positive charge from $\mathrm{Ag}^{+}$

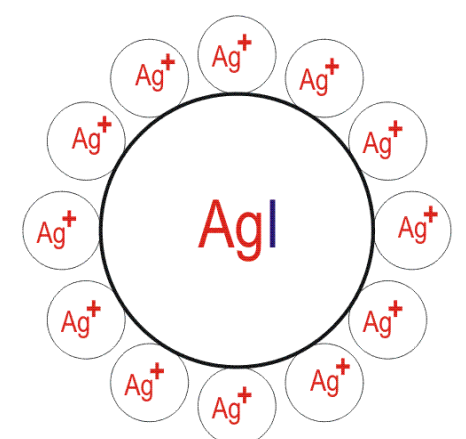

Negative charge from $\mathrm{I}^{-}$

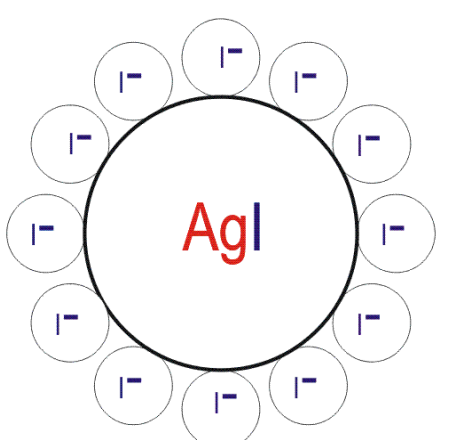

no charge

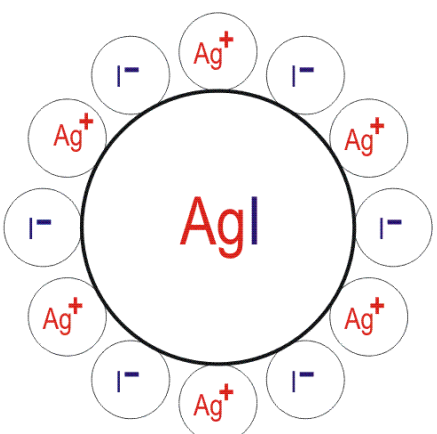

Figure 1. Schematic of charged silver iodide colloids showing a positive charge from silver ions, a negative charge from iodide ions, and a neutral colloid showing a random arrangement of the two ions. The simplified view shows the first layer and not the counter-ions to make up the double layer.

The objective here is to produce AgI with a similar, but opposite charge at two different AgI concentrations and determine the antimicrobial activity. To increase the exposure of AgI, an amorphous calcium phosphate (ACP) will be synthesized as a carrier phase and agent for bone regeneration. This work will report on the inclusion AgI during synthesis of ACP, the solubility of different ACP - AgI formulations, the silver release rate and the antimicrobial activity.

\section{Methods}

Synthesis. Silver iodide nanoparticles were synthesized using a condensation reaction route from silver nitrate and potassium iodide solutions: $\mathrm{AgNO}_{3}+\mathrm{KI}=\mathrm{AgI}+\mathrm{KNO}_{3}$. Silver iodide is an ionic crystal, and the surface charge arises from an excess of ionically bonded constituent ions [1]. A $0.01 \mathrm{M}$ solution of $\mathrm{AgNO}_{3}$ was added dropwise to a rapidly stirred $0.01 \mathrm{M} \mathrm{KI}$ solution. Charged $\mathrm{AgI}$ sols were formed by changing the solution concentrations. The $\xi$ potential changed from $-46 \mathrm{mV}$ to $+49 \mathrm{mV}$ and particle size varied from $33 \mathrm{~nm}$ to $51 \mathrm{~nm}$ for colloidal $\mathrm{AgI}^{-}$(negatively charged) and $\mathrm{AgI}^{+}$(positively charged) sols.

Amorphous calcium phosphate sols were produced by pouring a calcium nitrate solution into a stirred ammonium hydrogen phosphate solution buffered to $\mathrm{pH} 10$ using ammonium hydroxide so as to provide a $\mathrm{Ca} / \mathrm{P}$ ratio of 1.7 . The $\mathrm{ACP}$ was stirred for 10 minutes before modification by the silver iodide. Silver iodide was added with a positive or negative charge so as to substitute $5 \%$ and $10 \%$ of the calcium ions. Stirring was conducted for 15 minutes and the silver iodide combined with ACP was kept for 20 minutes before vacuum filtration. The filtrate was washed with water by pouring water onto the filter cake during vacuum filtration and then dried by freeze-drying.

Characterization and testing. The synthesized samples were characterized by X-ray powder diffraction (XRD), Fourier transform infrared spectroscopy (ATR-FTIR) and scanning electron microscopy (SEM). The XRD studies were performed on a Rigaku miniFlex II diffractometer operating with $\mathrm{Cu} K \alpha 1$ radiation. The data were collected over a $2 \theta$ range of $10^{\circ}$ to $70^{\circ}$ with a $0.01^{\circ}$ step size and $0.06 \mathrm{sec}$ per step. IR spectroscopy was used to analyze the bond interactions in the samples. Infrared ATR spectra from 400 to $4000 \mathrm{~cm}^{-1}$ were obtained using FT-IR spectrometer (Frontier FT-IR, Perkin Elmer). Evaluation of the microstructure and EDX analysis were performed with an ultra-high-resolution analytical FE-SEM (SU70 with EDS and EBSD and turbo-sputtering, Hitachi).

To determine the solubility, the powder was immersed in deionized water (electroconductivity $<$ $0.05 \mu \mathrm{S} / \mathrm{cm})$. Silver iodide modified amorphous calcium phosphates $(50 \mathrm{mg})$ were immersed in $5 \mathrm{~mL}$ of water for 48 and 96 hours at $37{ }^{\circ} \mathrm{C}$. After 48 hours, $5 \mathrm{ml}$ aliquots of solution were removed, and 5 $\mathrm{ml}$ of deionized water replenished in the test wells. The water samples remained clear. Silver release from silver-doped ACP was measured using a 180-50 Hitachi atomic absorption spectrometer (AAS) with an air-acetylene flame. Standard solutions of inorganic silver salts were used to calibrate the silver concentration. 
Antimicrobial testing. Antibacterial activity was estimated against two bacterial strains that are typical wound pathogens: Staphylococcus aureus MSCL 334 (Gram-positive bacterium) and Pseudomonas aeruginosa MSCL 331 (Gram-negative bacterium). Minimum inhibitory concentrations against test bacterial strains were determined by a standardized microdilution method using Muller-Hinton broth medium [2]. Experiments establish the antibacterial activity of each powder suspended in water after 24 hours.

\section{Results and Discussion}

The calcium phosphate phase stability was affected by the time of silver iodide addition. When the silver iodide was mixed with the calcium nitrate and then added to the phosphate solution, a crystallized tricalcium phosphate formed instead. To maintain an ACP, the silver iodide was then added separately to maintain the highly soluble ACP phase. X-ray diffraction of the dried sol showed the presence of both the AgI and the ACP; seen by the narrow $\beta$-AgI peaks (pdf \#0090374), and the broad ACP peak, Figure 2. Both negatively and positively charged sols could be added without disrupting the stability of the ACP. The FTIR spectra show broad phosphate peaks at $588 \mathrm{~cm}^{-1}$ and $1001 \mathrm{~cm}^{-1}$, corresponding to ACP, Figure 2. Silver iodide has no absorption within the spectra range of $800-4000 \mathrm{~cm}^{-1}$, and therefore was not detected in the FTIR spectra [3]. Carbonate is noted at $1421 \mathrm{~cm}^{-1}$ and $1490 \mathrm{~cm}^{-1}$. A similar XRD pattern and the same spectral features were observed for the 5\% and 10\% AgI enriched ACP.
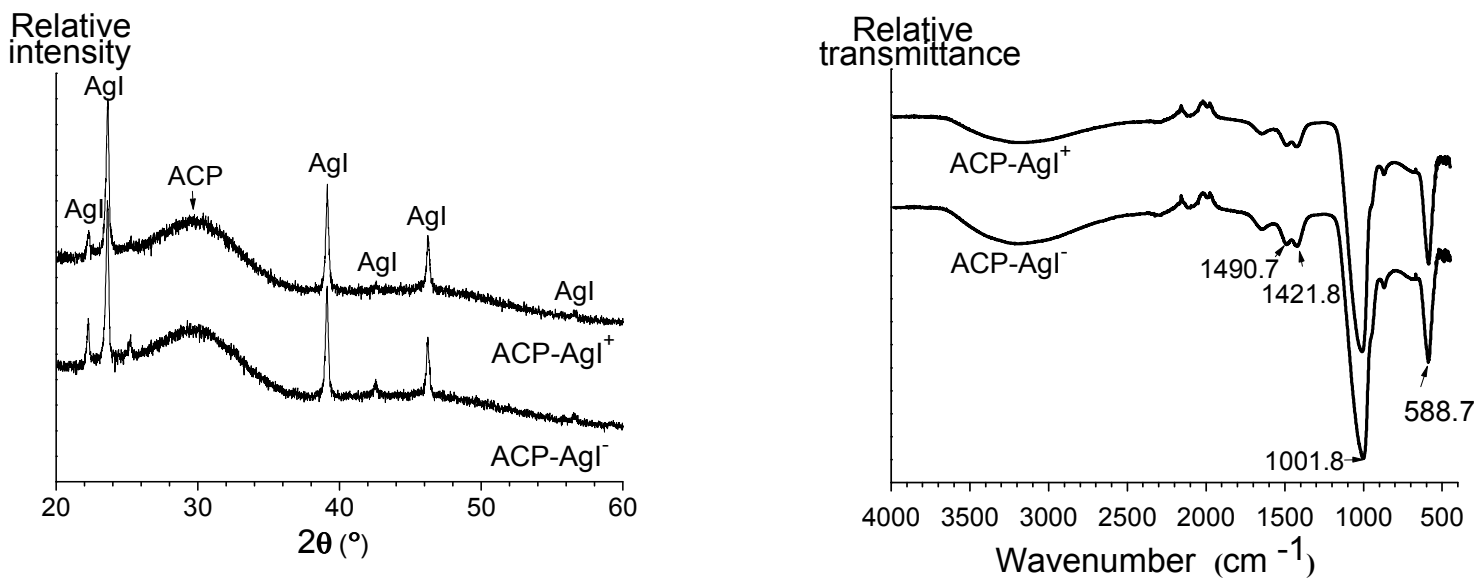

Figure 2. X-ray diffraction shows both $\beta$-AgI and amorphous calcium phosphate (ACP), while Fourier transform infra-red spectroscopy displays ACP from featureless absorption peaks.

Silver iodide was also inferred from silver in the dried sol, as measured by atomic absorption spectrometry. The silver content was about $0.2 \%$ for both compositions made from the negatively charged sols, $0.1 \%$ for the ACP $-5 \% \mathrm{AgI}^{+}$and $0.16 \%$ for the $\mathrm{ACP}-10 \% \mathrm{AgI}^{+}$powders. Silver and iodide was also detected using EXAFS, but since the silver iodide distribution is not necessarily homogeneous, the measurement of the concentration was not deemed sufficiently accurate. Dissolution of powder containing both $\mathrm{AgI}$ and $\mathrm{ACP}$ analyzed a larger powder volume than the electron-beam in the scanning electron microscope.

The microstructure was similar for both the formulations containing AgI; ACP particles were about $50-100 \mathrm{~nm}$ for the powder containing $\mathrm{AgI}^{-}$, but smaller for the $\mathrm{ACP}-\mathrm{AgI}^{+}$, Figure 3. Silver iodide appeared uniformly distributed, but was also found as agglomerates (not shown here). 

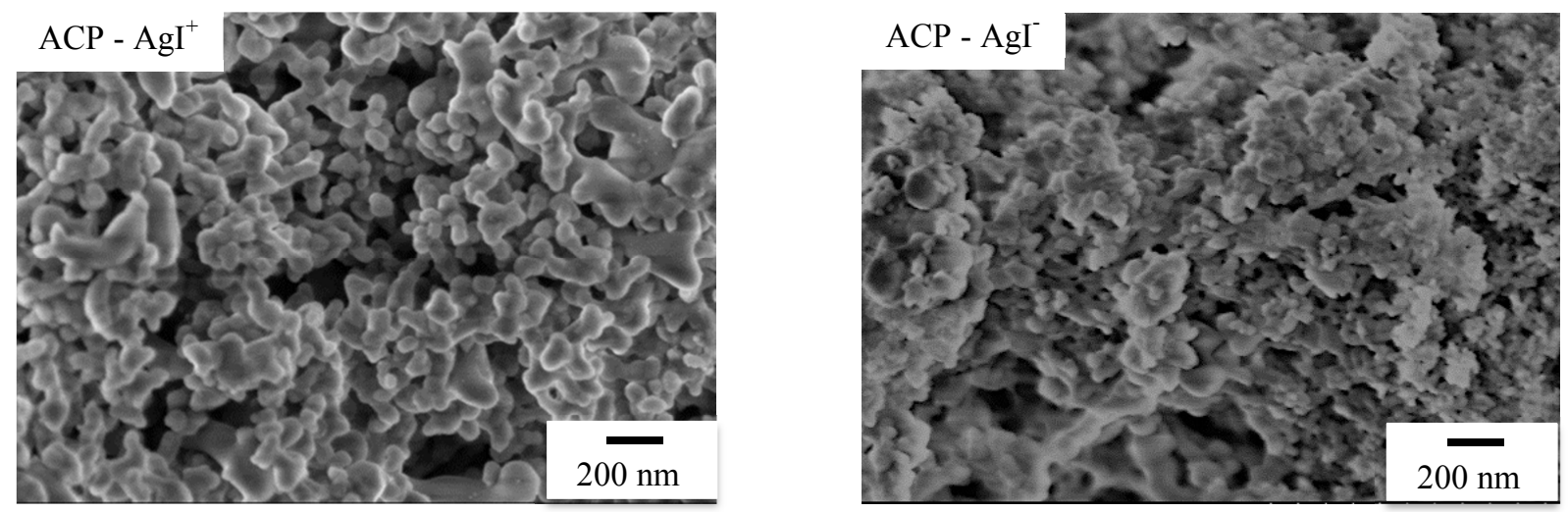

Figure 3. The microstructure of ACP - $\mathrm{AgI}^{+}$and ACP - AgI powders seen in the SEM.

The intention is for dissolution of the ACP - AgI to provide the necessary ions for bone regeneration and either releasing AgI nanoparticles or silver and iodide for antimicrobial action. The dissolution of the calcium phosphates coupled with AgI increased the $\mathrm{pH}$ for all formulations. Immersion of ACP into deionized water increased the $\mathrm{pH}$ from 6.3 to 6.6, Table 1. When AgI was present, the $\mathrm{pH}$ was always higher; a greater $\mathrm{pH}$ exhibited by the AgI that was initially prepared with a negative charge before addition to the ACP sol. Immersion of freeze dried ACP - AgI into deionized water also showed a high silver release in the same time period. Very little change in the release of silver was found after the second $48 \mathrm{~h}$ immersion in fresh deionized water.

The decrease in the release of silver during the second $48 \mathrm{~h}$ period may arise from a more stable layer on the ACP. This would require examination of the powder after $48 \mathrm{~h}$ to determine the changes on the surface.

Table 1. Dissolution of ACP - AgI measured by $\mathrm{pH}$, and the silver release after 48 and $96 \mathrm{~h}$.

\begin{tabular}{|l|l|l|l|}
\hline & \multicolumn{2}{|c|}{$\mathbf{p H}$} & \multicolumn{2}{|c|}{ Released Ag+ } \\
\cline { 3 - 4 } & $($ after 48h) & $\%$, after $48 \mathrm{~h}$ & $\%$, after 96h \\
\hline Deionized $\mathrm{H}_{2} \mathrm{O}$ & 6.28 & - & - \\
\hline $\mathrm{ACP}$ & 6.55 & 0 & 0 \\
\hline $\mathrm{ACP}-5 \% \mathrm{AgI}^{-}$ & 7.22 & 0.042 & 0.043 \\
\hline $\mathrm{ACP}-10 \% \mathrm{AgI}^{-}$ & 7.59 & 0.046 & 0.049 \\
\hline $\mathrm{ACP}-5 \% \mathrm{AgI}^{+}$ & 7.26 & 0.001 & 0.002 \\
\hline $\mathrm{ACP}-10 \% \mathrm{AgI}^{+}$ & 6.36 & 0.002 & 0.003 \\
\hline
\end{tabular}

A clear difference was seen between formulations made from ACP and positively or negatively charged AgI particles. The positively charged AgI did not inhibit bacteria growth, Table 2. A comparison to ACP shows no difference. Negatively charged AgI inhibit Pseudomonas aeruginosa growth at concentrations of $\geq 0.3-0.6 \mathrm{mg} / \mathrm{ml}$. This is important since this type of bacteria thrives on most surfaces and is commonly found on medical equipment where it can cause cross-infections in hospitals. Staphylococcus aureus also is found on surfaces, but at least twofold higher concentrations of negatively charged AgI are required to inhibit bacterial growth.

The negative charge on outer cell envelope from the ionized phosphoryl and carboxylate substituents may interact with charged species in the surrounding environment [4]. The details regarding the interaction with the negatively charged bacteria surface need further investigations.

Since the silver release from the negatively charged AgI was significantly higher (see Table 1), it is not absolutely clear whether it is the larger dissolved silver and iodide concentration or the surface charge that gives the antimicrobial effect. Regardless of the mechanism, it is clear that ACP with negatively charged $\mathrm{AgI}$ is more effective at inhibiting bacteria growth. 
Table 2. The Minimum Inhibitory Concentration (MIC) and the Minimum Bactericidal Concentration (MBC) in $\mathrm{mg} / \mathrm{ml}$ for differently charged AgI nanoparticles with ACP.

\begin{tabular}{|l|c|c|c|c|c|c|}
\hline \multirow{2}{*}{ Powder } & \multicolumn{3}{|c|}{$\begin{array}{c}\text { Pseudomonas aeruginosa } \\
\text { MSCL 331 (Gram-negative) }\end{array}$} & \multicolumn{3}{c|}{$\begin{array}{c}\text { Staphylococcus aureus } \\
\text { MSCL 334 (Gram-positive) }\end{array}$} \\
\cline { 2 - 7 } & MIC & MBC & MBC/MIC & MIC & MBC & MBC/MIC \\
\hline Blank - ACP & $>10$ & $>10$ & & $>10$ & $>10$ & \\
\hline ACP - 5\%AgI & 0.3 & 2.5 & 8.3 & 1.3 & 10 & 7.7 \\
\hline ACP - 10\%AgI & 0.6 & $>10$ & $>16$ & 1.3 & $>10$ & $>7.7$ \\
\hline ACP - 5\%AgI & $>10$ & $>10$ & & $>10$ & $>10$ & \\
\hline ACP - 10\%AgI & $>10$ & $>10$ & & $>10$ & $>10$ & \\
\hline
\end{tabular}

The minimum bactericidal concentration (MBC) that kills all bacteria is greater than the MIC. It is seen that the $5 \% \mathrm{AgI}^{-}$composition is more capable in not only preventing growth, but also in killing the bacteria. This may be attributed to a larger surface area available for release. The particle size of $\mathrm{AgI}$ reported here is $33 \mathrm{~nm}$ got negatively charged $\mathrm{AgI}$ and $51 \mathrm{~nm}$ for positively charged AgI, however, a further reduction will be more effective.

Previous work has shown the limited incorporation of silver into the apatite lattice that has provided antibacterial activity for only the first two hours [5]. This shortfall is overcome here from the use of a more soluble ACP matrix that can release AgI particles to provide an antibacterial response over a longer period. If necessary, the bioactivity can be lowered by creating different degrees of crystallinity in the matrix. The next experiments will address the use of a crystalline matrix for comparison to the more soluble amorphous calcium phosphate matrix.

While silver phosphate nanoparticles in hydroxyapatite and silver doped hydroxyapatite is known to provide antibacterial activity [6,7], the effect is expected to be greater for AgI; both silver and iodide have antibacterial properties. This work has shown a new concept where the release of both silver and iodide from AgI enriched ACP inhibit bacterial survival.

\section{Summary}

Silver iodide sols were synthesized with a positive or negative charge, the extent of which was measured by the zeta potential. These were incorporated in amorphous calcium phosphate (ACP), characterized for phase composition, tested for solubility and analyzed for the bacterial response. Crystalline AgI nanoparticles were formed and were incorporated at similar concentrations in all powders. Amorphous calcium phosphate provided a high solubility matrix for increasing the bioavailability of AgI. The dissolution of AgI, measured by the release of silver, was significantly higher for negatively charged AgI than for positively charged AgI. The antibacterial activity was greatest for negatively charged AgI in both Pseudomonas aeruginosa and Staphylococcus aureus containing media.

Acknowledgements. The inclusion of negatively and positively charged AgI in ACP was conceived by DK and KAG. Experimental work was conducted by DK, ZS and VN. Gratitude is expressed to Martynas Misevicius from Vilnius University for the SEM images. The article was written by KAG, DK and ZS. All authors contributed to the interpretation of results and discussion, have corrected, read, and approved the final manuscript. The project was supported by the TaiwanLatvian-Lithuanian Foundation for Scientific Co-operation within the project "Nanoscaled functional materials for biotechnological and optical applications" (No. IZM 11-13-0501/21) and Riga Technical University. ZS was funded by the Research Council of Lithuania with a postdoctoral fellowship from the European Union Structural Funds project "Postdoctoral Fellowship Implementation in Lithuania". 


\section{References}

[1] H.H. Kolkmeijer, J.W.A. van Hengel, On regular and hexagonal silver iodide. Zeit. Krist. 88 (1934) 317-22.

[2] S. Qaiyumi, Macro- and microdilution methods of antimicrobial susceptibility testing. in: R. Schwalbe, L. Steele-Moore, A.C. Goodwin (Eds.), Antimicrobial susceptibility testing protocols. CRC Press, 2007, pp. 75-79.

[3] Q. Zhu, X. Su, H.J. Wu, Y.J. Zhai, J.M. Xia, Y.Z Xu, J.G. Wu, The analysis of silver iodide fine particles of TLC/FTIR matrix. Spectroscopy and Spectral Analysis, 32 (2012) 1790-94.

[4] W.W. Wilson, M.M. Wade, S.C. Holman, F.R. Champlin, Status of methods for assessing bacterial cell surface charge properties based on zeta potential measurements, J. Microbiol. Meth., 43 (2001) 153-64.

[5] O. Ivashchenko, L. Protsenko, O. Budylina, N. Ulianchich, T. Yarmola, Influence of silver doping on hydroxyapatite properties. Advanced Science, Engineering and Medicine, 5 (2013) 26674.

[6] J.J. Buckley, A.F. Lee, L. Olivi, K. Wilson, Hydroxyapatite supported antibacterial $\mathrm{Ag}_{3} \mathrm{PO}_{4}$ nanoparticles. J. Mater. Chem. 20 (2010) 8056-63.

[7] C.S. Ciobanu, S. L. Iconaru, P. De Coustumer, L.V. Constantin, D. Predoi, Antibacterial activity of silver-doped hydroxyapatite nanoparticles against gram-positive and gram-negative bacteria. Nanoscale Res. Lett. 7 (2012) 324. 
Bioceramics 25

10.4028/www.scientific.net/KEM.587

Charge State of Silver Halide Colloids Determines the Antibacterial Activity in Amorphous Calcium Phosphate

10.4028/www.scientific.net/KEM.587.74 